\title{
Human mirror neuron system responsivity to unimodal and multimodal presentations of action
}

\author{
Authors \\ Fran Copelli ${ }^{1}$, Joseph Rovetti ${ }^{1}$, Paolo Ammirante ${ }^{1}$, and Frank A. Russo ${ }^{1}$
}

\begin{abstract}
Affiliation
${ }^{1}$ Psychology Department, Ryerson University, Toronto, ON, Canada
\end{abstract}

Corresponding author

Frank A. Russo russo@ryerson.ca

\section{ORCID}

Copelli https://orcid.org/0000-0003-4538-6966

Rovetti https://orcid.org/0000-0002-9466-9607

Ammirante N/A

Russo https://orcid.org/0000-0002-2939-6358

\section{Acknowledgments}

We are indebted to Michael Schutz for early discussions regarding the experimental design. We thank Michael Zara for his work on the optical flow analyses and Miruna Ioan for assistance with data collection. We are also grateful to Makoto Miyakoshi and Seyed Yahya Shirazi for technical support with preprocessing of EEG data.

\section{Figures}

Figures were created using Adobe Photoshop, R, and MATLAB. 


\begin{abstract}
This study aims to clarify unresolved questions from two earlier studies (McGarry et al., 2012; Kaplan \& Iacoboni, 2007) on human mirror neuron system (hMNS) responsivity to multimodal presentations of actions. These questions are: (1) whether the two frontal areas originally identified by Kaplan and Iacoboni (ventral premotor cortex [vPMC] and inferior frontal gyrus [IFG]) are both part of the hMNS (i.e., do they respond to execution as well as observation), (2) whether both areas yield effects of biologicalness (biological, control) and modality (audio, visual, audiovisual), and (3) whether the vPMC is preferentially responsive to multimodal input. To resolve these questions about the hMNS, we replicated and extended McGarry et al.'s electroencephalography (EEG) study, while incorporating advanced source localization methods. Participants were asked to execute movements (ripping paper) as well as observe those movements across the same three modalities (audio, visual, and audiovisual), all while 64channel EEG data was recorded. Two frontal sources consistent with those identified in prior studies showed mu event-related desynchronization (mu-ERD) under execution and observation conditions. These sources also showed a greater response to biological movement than to control stimuli as well as a distinct visual advantage, with greater responsivity to visual and audiovisual compared to audio conditions. Exploratory analyses of mu-ERD in the vPMC under visual and audiovisual observation conditions suggests that the hMNS tracks the magnitude of visual movement over time.
\end{abstract}

\title{
Keywords
}

Mirror neuron system, EEG, independent component analysis, modality, biological movement 


\section{Introduction}

Scholars have long been interested in the mechanisms by which humans are able to understand the actions of others. Because of this, a great deal of excitement was generated from early single-cell recordings of the monkey premotor cortex (F5) and inferior parietal cortex (PF/PFG), which revealed comparable patterns of activity under action execution and action observation conditions (di Pellegrino et al., 1992; Fogassi et al., 2005; Gallese et al., 1996; Rizzolatti et al., 1996a; Rozzi et al., 2008; Yamazaki et al., 2010). These studies provided some of the first evidence suggesting the existence of "mirror neurons." While the existence of mirror neurons in humans remains an open question (but see Mukamel et al., 2010), a network of activity has been identified that appear to possess similar functionality (i.e., responsiveness to action execution and observation) regardless of whether putative mirror neurons are involved.

The human mirror neuron system (hMNS) is a fronto-parietal network proposed to be involved in the execution as well as the "simulation" of observed actions (Jeannerod, 2001), which may underpin action understanding (Gallese \& Goldman, 1998; Rizzolatti et al., 2001). Visual and multisensory information is thought to be projected to frontal and parietal nodes of the system via a dorsal route originating in the superior temporal sulcus (STS; Rizzolatti \& Craighero, 2004). The system has been shown to be more responsive to action that is biological, such as the movement of human hands, than non-biological action, such as the blinking of a square (Cochin et al., 1998; McGarry et al., 2012; Ulloa \& Pineda, 2007). More controversial causal claims regarding its involvement in perception of speech (Fadiga et al., 2002; Wilson \& Iacoboni, 2006) have been called into question (Hickok, 2010; Rogalsky et al., 2011).

Evidence for the hMNS has largely been obtained using non-invasive methods such as functional magnetic resonance imaging (fMRI; e.g., Chong et al., 2008; Kaplan \& Iacoboni, 2007), functional near-infrared spectroscopy (e.g., Brucker et al., 2015), transcranial magnetic stimulation (TMS; e.g., Fadiga et al., 2002; Naish et al., 2014), and electroencephalography (EEG; e.g., Alhajri et al., 2018; Babiloni et al., 2002; Perry \& Bentin, 2009). An obvious advantage of EEG is the potential to record the timing of brain responses to specific event onsets. For example, event-related desynchronization of EEG activity has been observed to lag postmotion onset in apparent motion stimuli by about 200-250 msec (Rossi et al., 2014). A few recent investigations of the hMNS have also incorporated electrocorticography (e.g., Kern et al., 2019; Perry et al., 2018), allowing for high temporal as well as spatial resolution. Collectively, these studies have demonstrated that the hMNS includes a frontal node composed of the ventral premotor cortex (vPMC) and the inferior frontal gyrus (IFG), and a parietal node located in the inferior parietal lobule (IPL). The frontal node is thought to be the homolog of monkey area F5 and the parietal node is thought to be the homolog of monkey area PF/PFG (e.g., Filimon et al., 2015; Gazzola \& Keysers, 2009).

Although most early research into the involvement of mirror neurons in perception concerned visual action, some research has also demonstrated their responsiveness to auditory action. The first example of this was Kohler et al. (2002), who studied mirror neurons in monkey area F5 and found that $16 \%$ of them responded when perceiving sounds associated with actions (e.g., ripping paper; crushing a peanut) but not when perceiving non-action sounds. Some of 
these neurons also responded to the visual counterparts to these actions. Keysers et al. (2003) further studied these "audiovisual mirror neurons" and found that $36 \%$ of them responded more to the audiovisual observation of actions than to the unimodal (i.e., visual or auditory) observation of actions. In other words, these neurons exhibited an additive response to audiovisual stimuli, one that is greater for multimodal stimuli than unimodal stimuli. Thus, it was proposed that this particular subset of mirror neurons may be involved in the perception of multimodal action, and more speculatively that these neurons may be indifferent to the modality of sensory input (i.e., amodal).

On the basis of this animal work, Kaplan and Iacoboni (2007) set out to investigate hMNS responsiveness to the same actions presented across different modalities. Participants perceived biological action stimuli (human hands ripping paper) and control stimuli (a blinking square accompanied by a pure tone) in audio, visual, and audiovisual presentation conditions. Consistent with Keysers et al. (2003), the fMRI results showed that a portion of the left vPMC responded more to audiovisual biological stimuli than to unimodal biological stimuli or control (non-biological) stimuli. Indeed, visual inspection of this area's activity suggests that it responds very little if at all to unimodal stimuli. Kaplan and Iacoboni suggested that this area may be yetto-be-defined portion of the left vPMC and proposed that this area may play a role in multisensory integration.

Kaplan and Iacoboni (2007) also identified bilateral anterior IFG sources that preferentially responded to biological sound over control sound. However, they acknowledged that these sources may not necessarily be a part of the classical hMNS. One possibility is that the anterior IFG regions identified by Kaplan and Iacoboni are instead responsible for semantic processing and associating sounds with meanings (see Bookheimer, 2002 for a review). Despite this, a more recent review suggested that anterior portions of the IFG may house neurons with mirror-like properties (Bonini, 2017). One limitation of Kaplan and Iacoboni's study is that it did not include an action execution condition (i.e., one in which participants ripped paper). As a result, it is unclear whether the regions that they identified are responsive to action execution, which further calls into question whether they possess features characteristic of the hMNS (Hobson \& Bishop, 2017).

McGarry et al. (2012) replicated and extended the findings of Kaplan and Iacoboni (2007) using event-related desynchronization of the mu rhythm (mu-ERD) as an EEG index of the hMNS. Participants were asked to observe the same paper-ripping hands and control stimuli used by Kaplan and Iacoboni. After subjecting EEG data to a blind-source separation procedure, a source was localized to be only $14 \mathrm{~mm}$ away from the portion of the left vPMC identified by Kaplan and Iacoboni. Consistent with the fMRI results, McGarry et al. found that activation of this area was greater in response to all biological action conditions than their control counterparts, and greater in response to audiovisual biological action stimuli than to unimodal biological action stimuli. However, McGarry et al. did not identify a source consistent with the anterior IFG.

McGarry et al. (2012) did include a paper-ripping action execution condition in order to test a fundamental assumption of the hMNS - that it would exhibit a similar response to action 
execution and action observation conditions. Results showed that the vPMC source did not respond to the execution of paper-ripping. Since the hMNS is theoretically defined by activation in response to both action observation and execution, this result questions whether this portion of the vPMC is indeed part of the hMNS. Several limitations of McGarry et al.'s study may have contributed to this unexpected finding. For instance, it used an EEG system with only 24 channels, it analyzed only the first $2.5 \mathrm{~s}$ of the 8-s stimuli, and it used the Second-Order Blind Identification (SOBI) procedure for blind source-separation - a procedure that is computationally efficient but less effective than other algorithms such as InfoMax (Delorme et al., 2007).

Some recent anatomo-functional evidence (Bruni et al., 2018) points to the intraparietal sulcus (IPS) as another structure that may be part of the core hMNS. It has been suggested that the STS - IPL - VPMC pathway, originally considered to be the main pathway of visual input to the premotor cortex, may be more precisely represented by an STS - IPS - VMPC pathway (Lanzilotto et al., 2019). This important role for the IPS (putative monkey homologue of anterior intraparietal area) is further supported by single unit recordings taken in posterior parietal cortex during observed actions (Aflalo et al., 2020; Lanzilotto et al., 2020).

Taken together, the finding of the two earlier studies on hMNS responsivity to multimodal presentations of paper-ripping lead to the following questions: (1) are the two frontal areas originally identified by Kaplan and Iacoboni (2007) reasonably considered part of the hMNS (i.e., do they respond to action execution), and (2) do both frontal areas yield effects of biologicalness (biological, control) and modality (audio, visual, audiovisual), and (3) is the vPMC preferentially responsive to multimodal input. The current study aimed to address the above questions. To achieve this aim, we replicated and extended McGarry et al.'s (2012) EEG study. Participants were presented with the same biological stimuli (paper-ripping) and control stimuli (a blinking square and pure tone) across three modalities (audio, visual, audiovisual), all while 64 channel EEG data was recorded. EEG data was subjected to InfoMax, a computationally exhaustive blind-source separation procedure, to identify independent components (ICs) corresponding to areas of interest. In addition to the conditions tested in the prior two studies, we included two types of incongruent stimuli to provide us with an opportunity to assess whether audiovisual facilitation occurs when the unimodal components of bimodal stimuli do not match one another.

\section{Methods}

\section{Participants}

Participants were recruited from the Ryerson University Psychology Research Pool. Of the 24 participants recruited, three were excluded for the following reasons: failing to complete the study $(n=2)$, and failing to understand the task $(n=1)$. The 21 participants with usable data included 13 females and eight males ranging in age from 18 to 32. The study was approved by the Research Ethics Board at Ryerson University. Participants involved in the study were provided with credit towards their introductory psychology courses. 


\section{Stimuli}

Each of the eight observation conditions was associated with a unique stimulus. Six of these stimuli (see Figure 1; conditions 1-6) were identical to those used by Kaplan and Iacoboni (2007) as well as McGarry et al. (2012). These six stimuli were produced by crossing two levels of biologicalness (biological, non-biological control) with three levels of modality (audio, visual, audiovisual). The biological stimuli consisted of audio and/or visual presentations of human hands ripping paper; the control stimuli consisted of a digital square that moved along a vertical plane in the same motion as the hands and/or a pure tone that was temporally aligned with the onset of paper rips. Pure tones were $261.7 \mathrm{~Hz}$ in frequency and 200-500 msec in duration, with a $15 \mathrm{msec}$ onset rise time and gradual decay (i.e., percussive envelope). Our study also included two exploratory incongruent audiovisual conditions that were constructed by combining portions of the original stimuli as follows: biological audio with control visual, and control audio with biological visual (see Figure 1; conditions 7 and 8). All stimuli were $8 \mathrm{~s}$ in duration, and presented with a 5-s interstimulus interval and 1.5-s baseline.

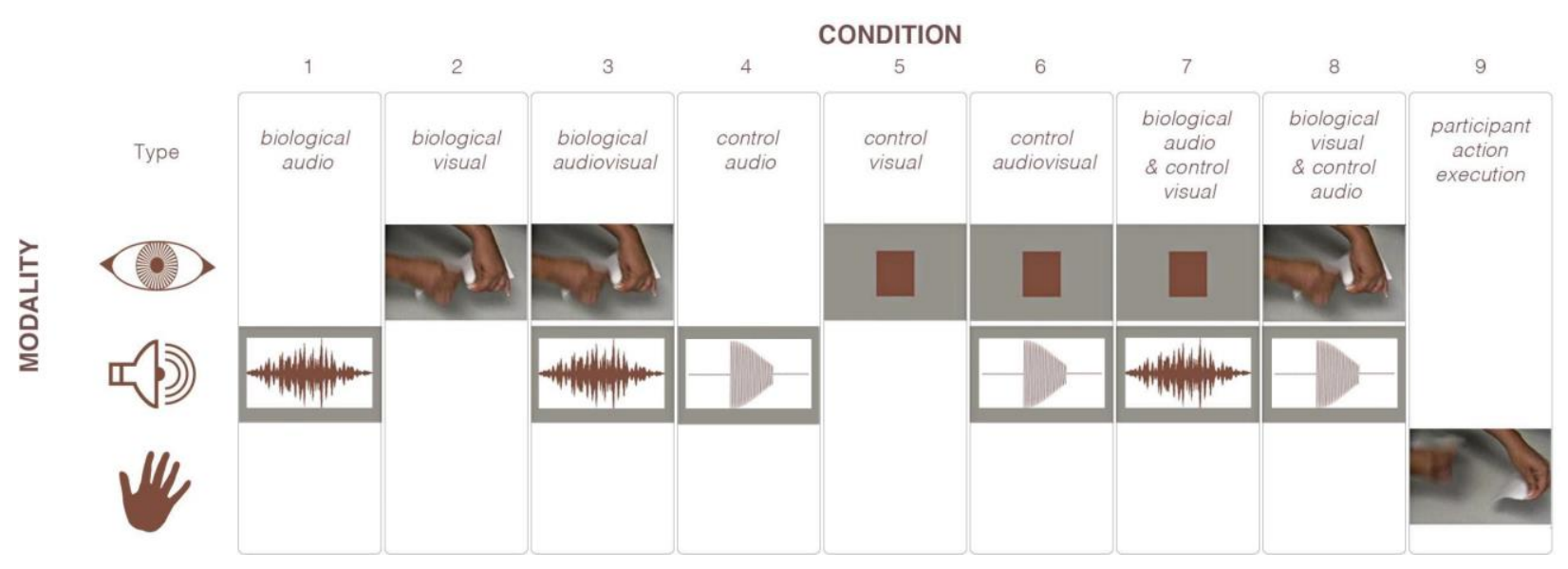

Fig. 1. Depiction of the eight observation conditions and the one execution condition. The stimuli used in the first six observation conditions were identical to those used by Kaplan and Iacoboni (2007) as well as McGarry et al. (2012). The stimuli used in the other two observation conditions were novel to the current study, involving incongruent pairings of audio and visual stimuli.

\section{Procedure}

After completing an informed consent, participants were brought inside an Industrial Acoustics Company sound-attenuated chamber where they were seated in a chair that was $60 \mathrm{~cm}$ away from a visual display flanked by two loudspeakers. Participants were capped with a Biosemi 64-channel ActiveTwo EEG system using the International 10-20 System for electrode placement. The experimental stimuli were presented using a Windows desktop computer running Presentation ${ }^{\circledR}$ software (version 18.0, Neurobehavioral Systems, Inc., Berkeley, CA, www.neurobs.com). There were 20 repetitions of each of the nine conditions, equaling 180 trials in total. For every participant, these 180 trials were uniquely randomized and presented in four equal 45 -trial blocks, with programmed breaks between each block.

Participants were asked to stay as still as possible during observation conditions, sustaining attention to the best of their ability. Similar to McGarry et al. (2012), during the action execution trials, participants were asked to rip a sheet of paper repeatedly until the end of the 8-s 
trial. Participants were encouraged to copy the actions observed in the cueing stimulus by ripping the paper sheet six times, but some variability was considered acceptable as long as participants were continually ripping the paper. The other eight conditions were passively perceived by participants, using a combination of audio and visual representations of the biological and control stimuli. Participants were able to anticipate which trial was coming next on the basis of the color of the fixation cross that appeared during the baseline; white indicated an action observation trial, and green indicated an action execution trial. Afterwards, participants were debriefed on the purpose of the study and allowed to ask any questions.

\section{Mu Rhythm}

The mu rhythm is typically defined as alpha band $(8-13 \mathrm{~Hz})$ activity generated over sensorimotor brain areas (central electrodes; e.g., C3, Cz, C4). This band is sometimes further subdivided into upper mu $(10-13 \mathrm{~Hz})$ and lower $\mathrm{mu}(8-10 \mathrm{~Hz})$. When source localization procedures are employed, EEG activity in the mu rhythm tends to cluster into a frontal node and a parietal node. As such, the event-related desynchronization that is observed over central electrodes during observation of intentional action (mu-ERD) may simply be the result of a decoupling of oscillations within and across these two functional nodes of the hMNS. This desynchronization is a type of event-related spectral perturbation (cf. synchronization) and sometimes referred to as mu suppression. While its relevance to motor behavior predates research on "mirror neurons" (e.g., Kuhlman, 1978; Sterman et al., 1976), it is broadly considered to be an index of hMNS activity (e.g., Fox et al., 2016; Lepage \& Theoret, 2006; Muthukumaraswamy et al., 2004), and is used to assess the hMNS in our study.

\section{Data Analysis}

The EEG data was sampled at $512 \mathrm{~Hz}$ and analyzed in MATLAB R2019b using EEGLAB 19.1, a widely used open-source MATLAB toolbox (Delorme \& Makeig, 2004). After the raw BDF data file was imported, a high-pass filter (pop_eegfiltnew) was applied at $1 \mathrm{~Hz}$ to remove baseline drift, and a low-pass filter was applied at $60 \mathrm{~Hz}$ to remove line noise. Next, noisy channels were identified using pop_rejchan and then interpolated using pop_interp. For each participant, an average of one external channel was rejected and three scalp channels were interpolated. Noisy scalp channels were always interpolated on the basis of surrounding channels; however, noisy external channels were always rejected. Data were referenced to the average (pop_reref) and epoched with a 1.5-s baseline and an 8-s stimulus.

\section{Independent Component Analysis (ICA) and Source Localization.}

After subtracting the baseline from the epoched data, an ICA was performed using the EEGLAB runica function (InfoMax algorithm). An ICA identifies unique sources of spectrotemporal variability in each participant's EEG data. When computing an ICA, the data provided must not be rank-deficient, otherwise, it results in "ghost" components, which is a result of insufficient information for the model. To avoid rank-deficient data, each participant's ICA yields $n$ number of components where $\mathrm{n}$ is equal to the number of electrodes less all interpolated channels. An initial ICA was used to isolate artifactual components with non-stereotyped activity. Next, noisy epochs were rejected per participant (at an average of 9\%) using default 
EEGLAB epoch rejection parameters. This step served the purpose of removing endogenous activity that was present prior to the stimulus.

Next, a second ICA was used to capture stereotyped neuro-electric activity (e.g., brain sources and oculomotor activity) leading to the final ICA weight matrixes for dipole fitting. The DIPFIT plugin was then used to locate dipoles using the boundary element model. Next, a plugin called IClabel (Pion-Tonachini et al., 2019) was used to perform automated independent component selection. At this point, we included a custom-written EEGLAB script (Miyakoshi, 2017) to perform automated component selection and reject independent components with more than $15 \%$ residual variance. An average of $5 \%$ of components were rejected per participant.

\section{Clustering and Event-Related Spectral Perturbations (ERSP)}

ICs were fit into clusters using the k-means algorithm (MacQueen, 1967), which, in this case, worked out to an average of one component per participant per cluster (SCCN, 2014). As we were only interested in changes in spectral power that occur as a result of the mu rhythm in hMNS areas (vPMC/IFG and IPL), clusters were formed using ERSPs and dipole location as our determining factors. Outlier components were classified as over three standard deviations from the mean, and were separated out. The resulting data was fit into 11 source clusters.

When analyzing the clusters, we considered their power spectrum as well as the estimated centroid of their dipoles. We entered their 3-D coordinates into Talairach Client version 2.4.3 to verify the locations in the brain (Talairach \& Tournoux, 1988). After identifying the two clusters whose dipole centroids were clearly locate in hMNS areas, we performed a time-frequency analysis and compared ERSPs in the $8-13 \mathrm{~Hz}$ and $0-8000 \mathrm{msec}$ range between experimental conditions. The first of these was the left vPMC, contained within the left precentral gyrus (BA 6), and the second was the left anterior IFG (BA 47). As may be seen in Figure 2, there was minimal overlap in the distribution of ICs across these two frontal clusters. Although none of the 11 clusters were spatially congruent with parietal regions of the hMNS, we did identify a nearby cluster in the right parietal lobe (right SPL). ERSP values associated with each cluster were collapsed across frequency $(8-13 \mathrm{~Hz})$ and time $(0-8000 \mathrm{msec})$ to acquire the mean power for each experimental condition. Finally, ERSP data was exported to CSV files using a customwritten MATLAB script.

\section{Statistical Analyses}

Repeated-measures analysis of variance (rmANOVA) was conducted for each cluster CSV file using the "ez" package in R version 3.6.1 (R Core Team, 2019), with GreenhouseGeisser adjustment of degrees of freedom applied to any effects that failed Mauchly's test of sphericity. Pairwise t-tests were done with Holm-Bonferroni correction for multiple comparisons (Holm, 1979), and the calculation of effect sizes for t-tests was done using the "Isr" package. In all statistical analyses, the source of error is between components rather than between participants. 


\section{Results}

\section{Left vPMC Cluster}

Figure 2a plots the centre Talairach coordinate (TAL -51, -9, 26) of the left vPMC cluster based on 21 independent components, contributed by 14 participants. This is likely the same brain area that was identified by Kaplan and Iacoboni (2007) as well as McGarry et al. (2012), as it is only $15 \mathrm{~mm}$ away from the region of interest in the former study and only $10 \mathrm{~mm}$ away from the cluster identified in the latter study. A preliminary one-sample t-test confirmed that mu-ERD in this cluster was significantly different from zero during the action-execution condition $(\mathrm{M}=-$ 2.24, $\mathrm{SD}=1.57), \mathrm{t}(20)=-6.53, \mathrm{p}<.0001, \mathrm{~d}=1.43$.
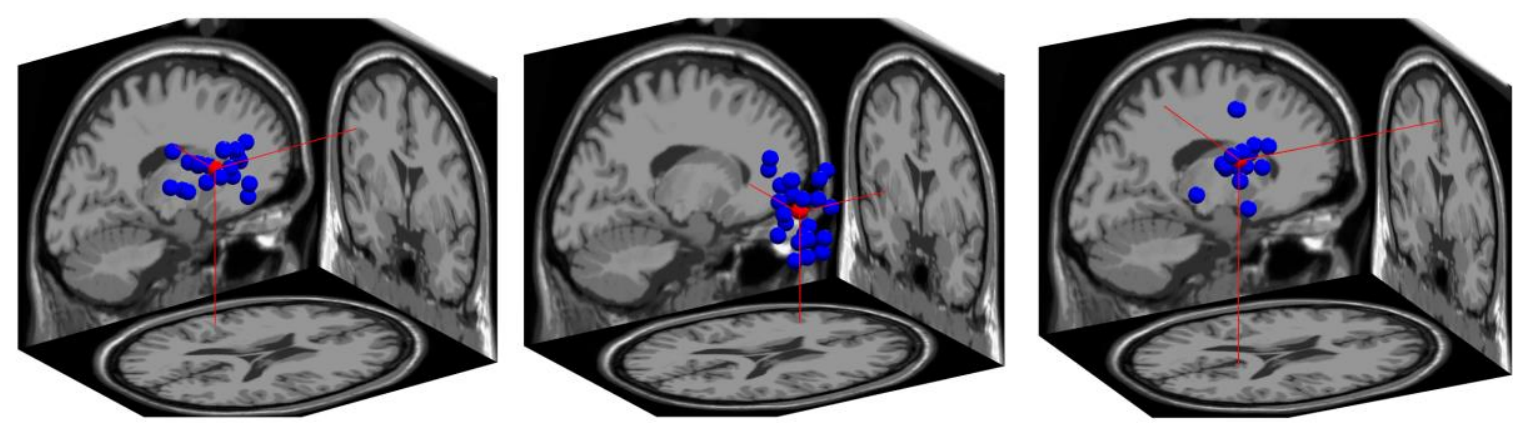

Fig. 2. Depiction of contributed components for each cluster of interest; note that not all participants contributed an IC to these clusters. (a) Centre (in red) and ICs (in blue) of the dipole cluster (TAL -51, -9, 26) located in BA 6, left vPMC; (b) Centre (in red) and IC's (in blue) of the dipole cluster (TAL -37, 30, -4) located in BA 47, left IFG (c) Centre (in red) and IC's (in blue) of the dipole cluster (TAL 4, -39, 54) located in BA 5, right SPL.

Figure 3 shows the effects of biologicalness and modality on mu-ERD during action observation in the left vPMC. A 2 (biologicalness) $\times 3$ (modality) rmANOVA revealed a significant main effect of biologicalness, $\mathrm{F}(1,20)=40.0, \mathrm{p}<.0001, \eta \mathrm{G} 2=.044$, with greater muERD for biological than control stimuli. In addition, there was a significant main effect of modality, $F(2,40)=14.7, p=.0009, \eta \mathrm{G} 2=.072$, with less mu-ERD for auditory compared to visual, $\mathrm{t}(41)=-4.63, \mathrm{p}=.0001, \mathrm{~d}=0.71$, or audiovisual, $\mathrm{t}(41)=-4.63, \mathrm{p}=.0001, \mathrm{~d}=0.71$. These main effects were qualified by a significant interaction between biologicalness and modality, $\mathrm{F}(40)=9.98, \mathrm{p}=.0003, \eta \mathrm{G} 2=.014$, which was driven by the greater effect of biologicalness in the visual and audiovisual conditions, $\mathrm{ds}=1.25$, than in the auditory condition, $\mathrm{d}=0.23$. Indeed, although the means were numerically in the predicted direction, the auditory condition was the only modality in which mu-ERD was not significantly greater in response to biological stimuli than in response to control stimuli. A follow-up analysis focusing on the first $2.5 \mathrm{~s}$ following stimulus onset (after McGarry et al., 2012) also failed to reveal the expected effect of biologicalness, $\mathrm{t}(20)=-0.85, \mathrm{p}=.41$. Because no audiovisual facilitation was found, the two exploratory (incongruent) conditions were not analyzed. 


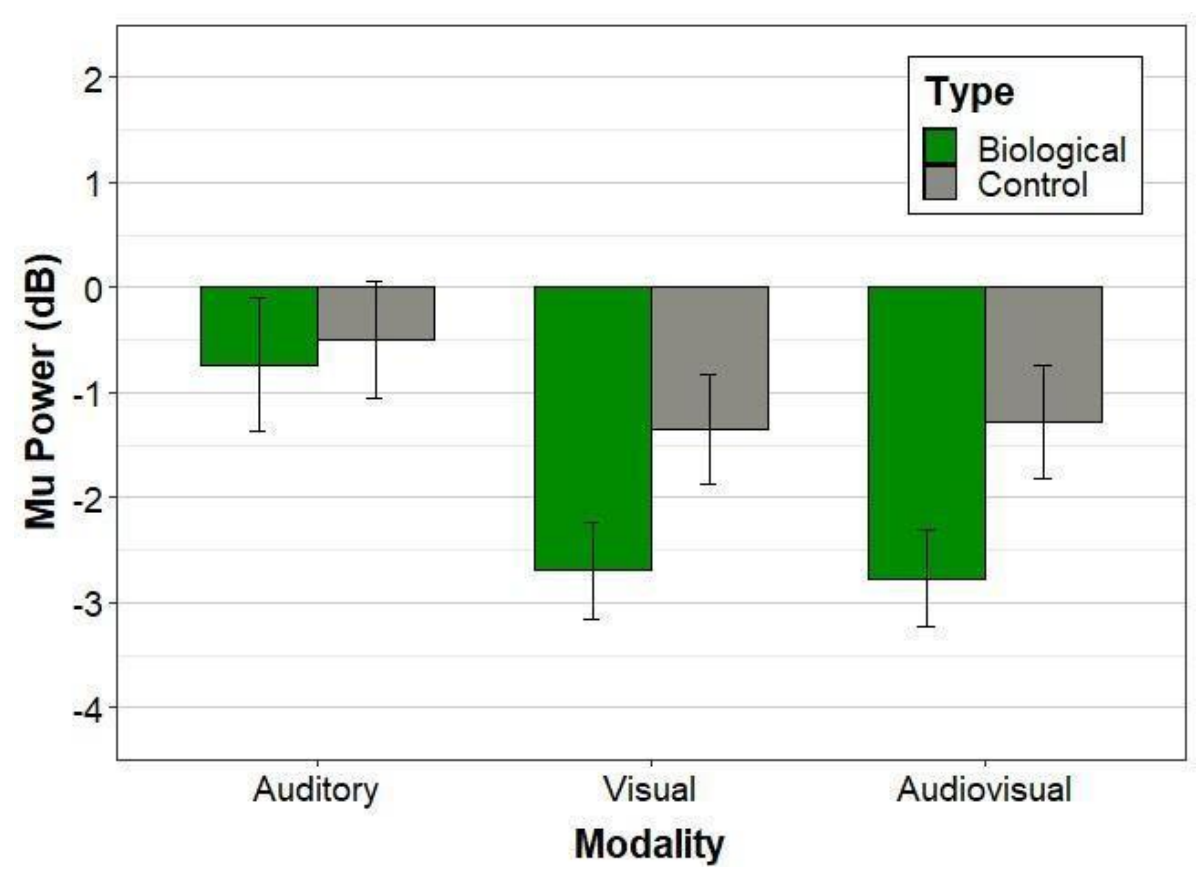

Fig. 3. The effects of biologicalness and modality on mu-ERD in the vPMC (BA6). There was a significant main effect of biologicalness on mu-ERD, such that mu-ERD was greater in response to biological stimuli than control stimuli; a significant main effect of modality on mu-ERD, such that mu-ERD was greater in response to visual and audiovisual stimuli than audio stimuli; and a significant interaction between biologicalness and modality on mu-ERD, such that mu-ERD is only greater in response to biological stimuli than to control stimuli in the visual and audiovisual modalities.

\section{Left IFG Cluster}

Figure $2 b$ plots the centre of the left IFG cluster (TAL -37, 30, -4) based of 21 independent components, derived from 16 participants. This source may be the same IFG area that was identified by Kaplan and Iacoboni (TAL, -50, 38, 0). Although both sources are in rostral IFG, our source was slightly posterior. A preliminary one-sample t-test confirmed that $\mathrm{mu}-\mathrm{ERD}$ in our left IFG cluster was significantly different from zero during the action-execution condition $(\mathrm{M}=-1.95, \mathrm{SD}=1.18), \mathrm{t}(20)=-7.58, \mathrm{p}<.0001, \mathrm{~d}=1.66$.

Figure 4 shows the effects of biologicalness and modality on mu-ERD during action observation in the left IFG cluster (BA 47). A 2 (biologicalness) $\times 3$ (modality) rmANOVA revealed a significant main effect of biologicalness on mu-ERD, $\mathrm{F}(1,20)=46.0, \mathrm{p}<.0001, \eta \mathrm{G} 2$ $=.13$, with mu-ERD greater for biological than control stimuli. In addition, there was a significant main effect of modality on mu-ERD, $\mathrm{F}(2,40)=14.7, \mathrm{p}=.0008, \eta \mathrm{G} 2=.21$, with less mu-ERD to audio stimuli than to visual stimuli, $\mathrm{t}(41)=-4.96, \mathrm{p}<.0001, \mathrm{~d}=0.77$, or to audiovisual stimuli, $\mathrm{t}(41)=-4.62, \mathrm{p}<.0001, \mathrm{~d}=0.71$. These main effects were qualified by a significant interaction between biologicalness and modality on mu-ERD, $F(2,40)=15.6, \mathrm{p}<$ $.0001, \eta \mathrm{G} 2=.045$, which was driven by the greater effect of biologicalness in the visual and audiovisual conditions, ds $=1.34$ and 1.33 , than in the auditory condition, $\mathrm{d}=0.29$. As with the vPMC cluster, the auditory condition was the only modality in which mu-ERD was not greater in response to biological stimuli than in response to control stimuli. Although the means were numerically in the predicted direction, the auditory condition was once again the only modality in which mu-ERD was not greater in response to biological stimuli than in response to control 
stimuli. However, a follow-up analysis focusing on the first $2.5 \mathrm{~s}$ following stimulus onset (after McGarry et al., 2012) did reveal the expected effect of biologicalness, $t(20)=-3.44, p=0.003$. Because no audiovisual facilitation was found, the two exploratory (incongruent) conditions were not analyzed.

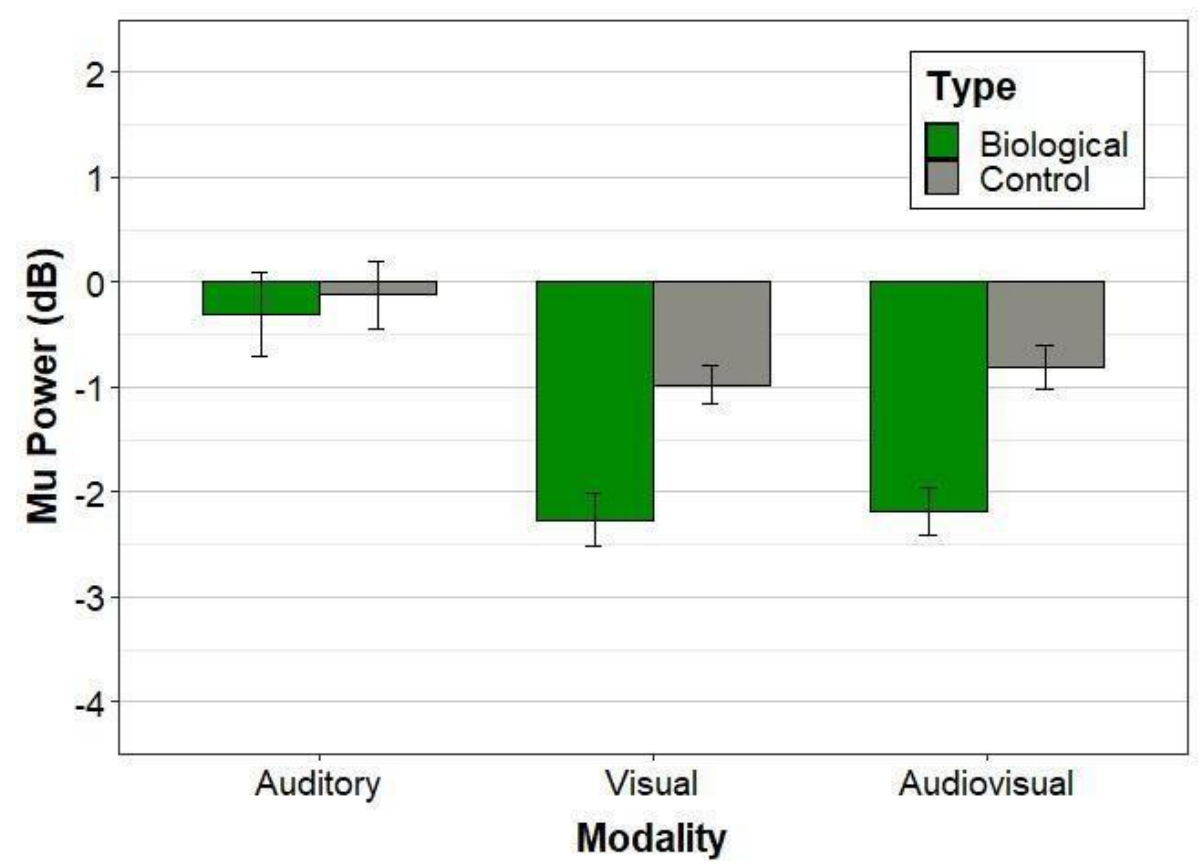

Fig. 4. The effects of biologicalness and modality on mu-ERD in the left IFG (BA 47). There was a significant main effect of biologicalness on mu-ERD, such that mu-ERD was greater in response to biological stimuli than control stimuli; a significant main effect of modality on mu-ERD, such that mu-ERD was greater in response to visual and audiovisual stimuli than audio stimuli; and a significant interaction between biologicalness and modality on mu-ERD, such that mu-ERD is only greater in response to biological stimuli than to control stimuli in the visual and audiovisual modalities.

\section{Right SPL Cluster}

The nearest source to the hMNS parietal node that the cluster analysis yielded was in the right SPL. Figure 2c plots the centre Talairach coordinate (TAL 4, -39, 54) of the right SPL cluster based on 17 independent components, contributed by 15 participants. The effects of biologicalness and modality in the SPL cluster were largely consistent with effects observed in the two frontal clusters. However, the cluster location was not theoretically implicated.

\section{Event-Related Spectral Perturbations (ERSP) of each cluster}

Figure 5 plots the ERSP data for sources across all six conditions. Although our planned analyses were conducted on mu-ERD values that were averaged across time and frequency, as is typical in EEG studies of the hMNS (Fox et al., 2016), the ERSP plots reveal distinct spectrotemporal variability, especially within the vPMC cluster for biological visual and audiovisual conditions. To further investigate this variability in the vPMC and whether it might be driven by the observation of movement dynamics, we generated three time series (Figure 6). The first time series was created by subjecting the biological visual stimulus to an optical flow analysis (Horn \& Schunck, 1981) using FlowAnalyzer (Barbosa et al., 2008). The algorithm computes pixel displacements between consecutive frames in video. All pixel displacements are 
represented as vectors representing magnitude and 2D direction. Those vectors are then summed into a single vector representing magnitude of movement over time. The second and third time series were created by collapsing mu-ERD across frequency in the vPMC cluster for the visual biological and audiovisual biological conditions. Each data point in these time series represented average power across frequencies for a given sample. All three time series were then resampled at $30 \mathrm{~Hz}$ and subjected to cross-correlation, which revealed a peak correlation when the mu-ERD time series lagged the magnitude of movement time series by $233 \mathrm{msec}(\mathrm{r}=-.81)$ in the visual biological condition and $200 \mathrm{msec}(\mathrm{r}=-.81)$ in the audiovisual biological condition (Figure 7).

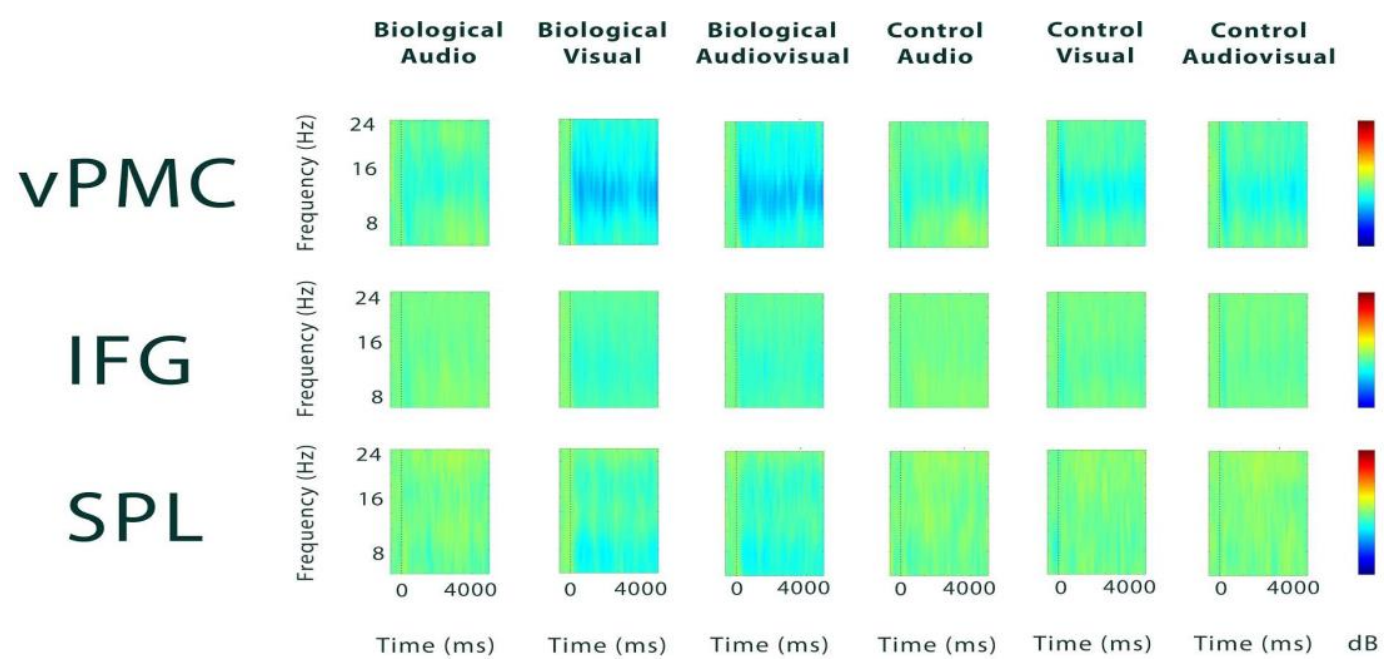

Fig. 5. ERSPs for the six replicated conditions in the vPMC source, IFG source, and SPL source. Time (-1000-6000 msec) is on the $\mathrm{x}$-axis and frequency $(8-25 \mathrm{~Hz})$ is on the $\mathrm{y}$-axis. Warmer colours are higher decibels $(\mathrm{dB})$ of power and cooler colours are lower.

\section{Magnitude of Motion \& ERSPs in the vPMC}
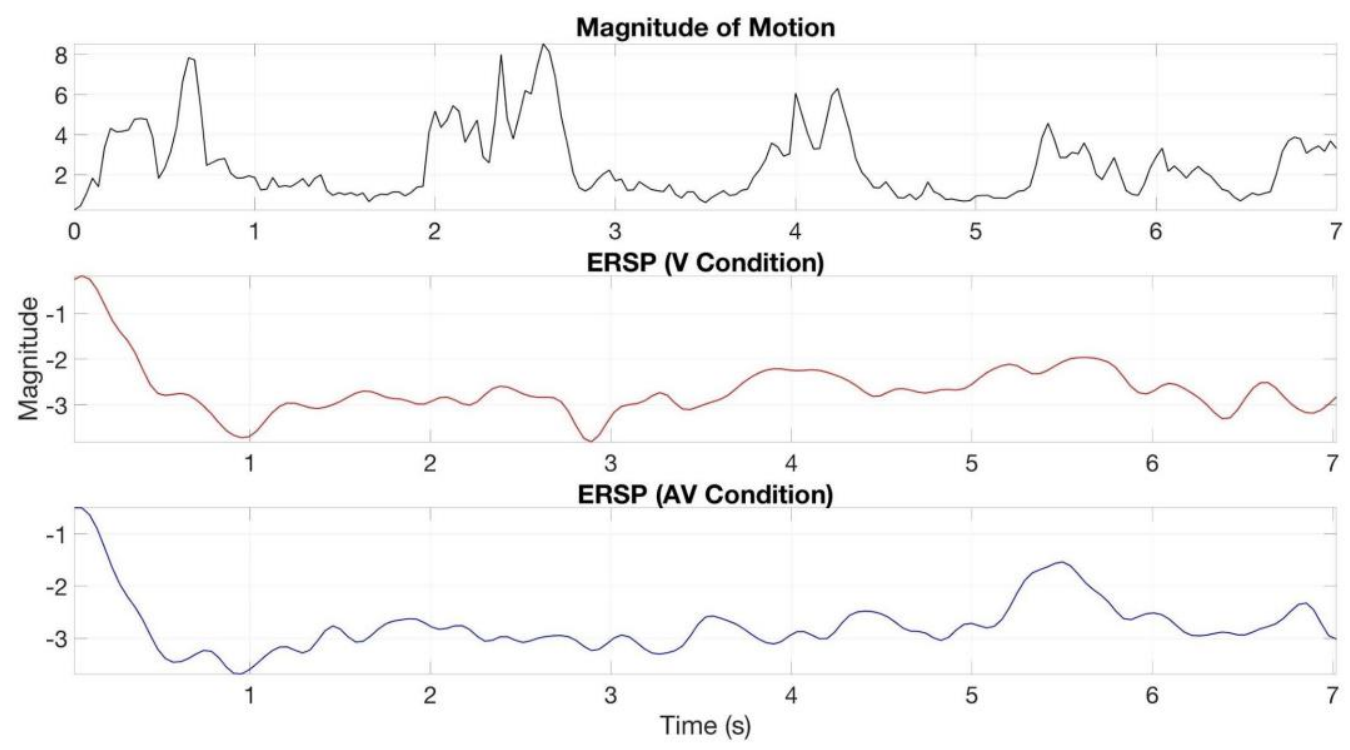

Fig. 6. Time series for: (a) magnitude of movement in the paper-ripping video, (b) mu-ERD in the vPMC for the visual biological condition; and (c) mu-ERD in the vPMC for the audiovisual biological condition. 

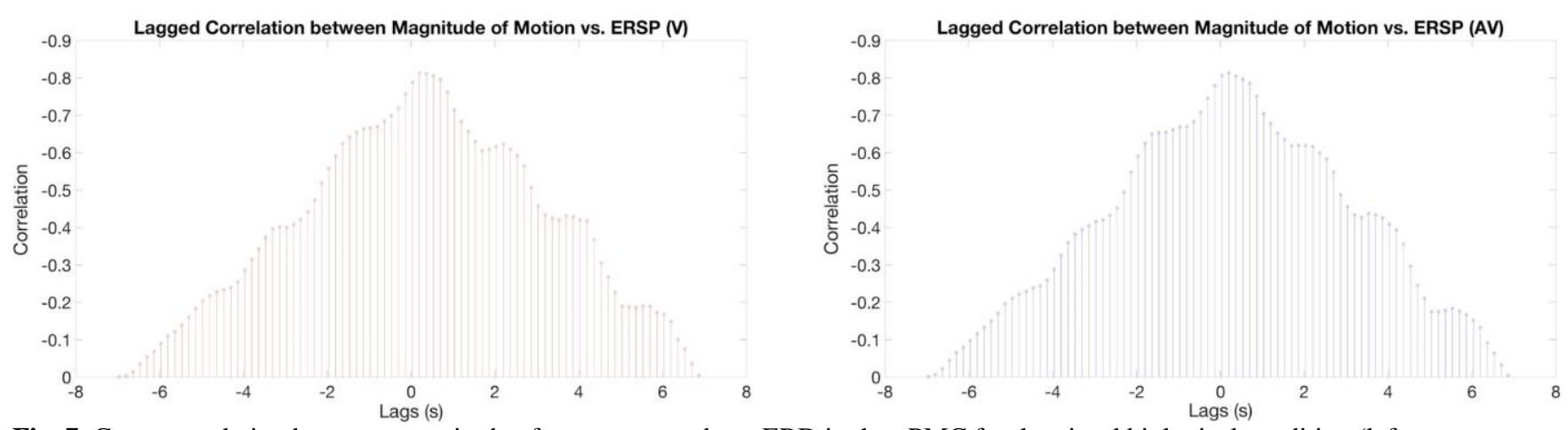

Fig. 7. Cross-correlation between magnitude of movement and mu-ERD in the vPMC for the visual biological condition (left panel); and mu-ERD in the vPMC for the audiovisual biological condition (right panel).

\section{Discussion}

The current study was a replication of McGarry et al. (2012), which was itself a replication of Kaplan and Iacoboni (2007). While there were some procedural differences across the studies, all used the same stimuli to investigate effects of biologicalness and modality of observed action on engagement of the hMNS. In the current study, we used a blind-source separation procedure to identify unique sources of brain activity. On the basis of the power spectrum and mean dipole location, two of the 11 sources identified were considered to be frontal hMNS candidates.

The frontal sources localized to brain areas that resemble sources found in two prior studies (Kaplan \& Iacoboni, 2007; McGarry et al., 2012). More specifically, our vPMC source (TAL -51, -9, 26) was likely the same source found by Kaplan and Iacoboni (MNI -64, 0, 18; TAL -61, -1, 18) and by McGarry et al. (TAL -57, -12, 18). Our left IFG source (TAL -37, 30, 4) bordered on Kaplan and Iacoboni's (2007) left IFG source (TAL, -50, 38, 0), and was contralateral to their right IFG source (TAL 52, 36, -8).

Much like the classic early studies of mirror neurons involving single-cell recordings in monkeys, the current study included an action-execution condition. Here, a positive finding supports our interpretation of these sources being part of the hMNS. The predicted finding was obtained with mu-ERD occurring in left IFG and left vPMC sources in response to both action execution and observation of biological action. Although a left vPMC source was also identified in the EEG study by McGarry et al. (2012), this earlier study did not observe mu-ERD for action-execution. Given the strong theoretically derived prediction for the present finding and its consistency across both sources, it would seem that McGarry et al.'s null finding was spurious. Consistent with findings from both prior studies using the same stimuli, we observed a biologicalness effect, wherein greater mu-ERD was obtained in the biological condition compared to the control condition (non-biological action). This biologicalness effect was observed in both frontal sources and is a hallmark of hMNS activity. So, what is it about biological action that differentiates it from non-biological action? Critically, our participants would have had experience executing movements like those depicted in our observation conditions but not like those in the control conditions. This lack of experience means that the 
stimuli would not have been able to activate a corresponding stored motor plan. Hence, it is not reasonable to expect that the hMNS would be called upon to support perception of action in the control condition. Prior studies that have utilized point-light displays of movement devoid of static cues have found evidence for activation of the hMNS (e.g., Saygin et al., 2004; Ulloa \& Pineda, 2007). However, in conditions where the point lights were scrambled or the movement removed (i.e., static point lights), the same activation effects were nullified. The current findings corroborate these point-light results and strongly suggest that biological movement is critical to perceptual engagement of the hMNS.

We observed a consistent visual advantage in both frontal sources with greater mu-ERD for visual and audiovisual compared to audio conditions. Unlike the two prior studies, we did not find any evidence for a multimodal enhancement in the vPMC source. Indeed, the mu-ERD for visual stimuli was not different from the mu-ERD for audiovisual stimuli. There are a few possible reasons for the discrepancy. First, it is important to note that although Kaplan and Iacoboni (2007) found evidence for audiovisual additivity in the vPMC, the area they identified using fMRI was spatially quite limited. Second, although McGarry et al. (2012) found this same result, they used a relatively sparse EEG montage (20 channels) and a source-localization procedure that is computationally efficient but less effective than other algorithms such as Infomax (Delorme et al., 2007). Hence, it seems possible that, although the vPMC sources identified across the three studies were consistent with respect to the Brodmann's areas implicated, the activity may not have overlapped completely. Given the mixed findings across studies, further research on the question of multimodal enhancement in the vPMC is warranted.

Auditory biological stimuli led to less mu-ERD than their visual and audiovisual counterparts in both sources. Moreover, the biologicalness effect was only evident after constraining the window of analysis to the initial 2.5 seconds following event onset and for the IFG source only. It is possible that the short-lived response has something to do with rapid neural adaptation known to take place in auditory cortex to repeated stimuli with consistent spectrotemporal variability (Dean et al., 2008). Over the initial 2.5 seconds, the participant would have been exposed to two presentations of a rip sound with near-identical spectrotemporal properties. Regardless of possible adaptation effects, evidence for attenuated response to auditory input can also be observed in the vPMC source identified by McGarry et al. (2012; see Figure 4). As such, we posit that the action simulation that occurs within the frontal hMNS is primarily driven by visual input. However, it is important to note that the stimuli used here (i.e., paper ripping) may not have been ideally suited to revealing consistent auditory engagement. While the auditory channel likely provided onset and acceleration cues, the visual channel would have also provided cues about grip strength, force, resistance, as well as the scale of movements.

Observing robust auditory engagement of the hMNS may require stimuli that carries salient information in the auditory channel that may support action understanding.

Speech is an example of a stimulus with salient auditory information that has been considered in relation to the hMNS (Fadiga et al., 2002; Wilson \& Iacoboni, 2006; Hickok, 2010). However, the perception of speech is not very well suited to elicit hMNS activity because of the rich semantic support provided by the ventral stream and the stereotypy of phonology in a 
given language. Better auditory candidates to consider would be perception of isolated syllables (Crawcour et al., 2009), vocal emotion (Rovetti et al., 2021), singing and/or music (Bagert et al, 2006; McGarry et al., 2015; Molnar-Szakacs \& Overy, 2006; Russo, 2020). These auditory stimuli are semantically abstract, which may give rise to compensatory involvement of the hMNS to support action understanding. Consistent with this view, Lévêque and Schön (2013) found greater mu-ERD for vocal (biological) compared with synthetic (non-biological) presentations of melodies. They also found that the mu-ERD was stronger in listeners who were less accurate singers, which is consistent with involvement of the hMNS as a compensatory mechanism. Along the same lines, Pruitt et al. (2019) found more automatic mimicry of laryngeal activity in less accurate singers during observation of auditory-only presentations of vocal melodies. Automatic mimicry is thought to be a downstream consequence of hMNS activity (Chartrand \& van Baaren, 2009).

As mentioned, the classical hMNS is proposed to have two nodes: a frontal node which may encompass two areas (vPMC and IFG), and a parietal node located in the inferior parietal lobule. Accordingly, it may appear somewhat unexpected that we were not able to identify a spatially-appropriate parietal source. Indeed, neither of the prior studies involving the stimuli used here found parietal activations. One proposal is that the frontal node supports a motor (afferent) simulation, whereas the parietal node supports a somatosensory (efferent) simulation (see Avenanti et al., 2007). It is possible that the act of paper ripping does not generate a clear somatosensory representation. Alternatively, it is possible that the motor simulation is sufficient to support action understanding.

Although both left frontal sources examined in this study were non-overlapping (see distribution of dipoles in Figure 2), they were not independent with respect to our experimental conditions (Figures 3 and 4 look strikingly similar). This lack of independence may give rise to concern about the source localization procedures used here. ICA procedures share a common assumption of independence of sources (inclusive of InfoMax and SOBI). This assumption is not realistic as separate nodes of a brain network are likely to have non-zero correlations. Hence, the orthogonalization process may force separate sources when only one truly exists (Vigario et al., 2000). However, it is important to highlight that the ERSP plots in Figure 5 minimize potential concerns about lack of independence. These plots reveal that the time-varying aspects of the muERD differed across sources even though the mean activity did not. For example, the desynchronization of activity observed in the vPMC source is largely constrained to upper mu $(10-13 \mathrm{~Hz})$, whereas it is evenly distributed across upper and lower mu $(8-10 \mathrm{~Hz})$ in the IFG source. Upper mu and lower mu have been functionally dissociated, with upper mu being tied to hand actions, and lower mu being somatotopically non-specific and tied to expectancy and attention (Cuellar et al., 2012; Pfurtscheller et al., 2000; Neuper, 2009).

A close examination of the ERSP plots in Figure 5 reveals modulation between strong and weak desynchronization in the vPMC source for the visual biological and audiovisual biological conditions. The rate of modulation appears to be a bit slower than once per second, which corresponds with the rate of paper ripping (6 rips over 8 seconds). This was an unexpected but intriguing finding that we sought to better understand through exploratory analyses. To do so, 
we generated three time series: (1) magnitude of movement in the paper-ripping video as obtained through an optical flow analysis; (2) magnitude of mu-ERD in the vPMC for the visual biological condition; and (3) magnitude of mu-ERD in the vPMC for the audiovisual biological condition. These time-series were subjected to cross correlation which yielded a negative correlation between magnitude of movement and the mu-ERD in the biological visual condition (peak $\mathrm{r}=-.81$ at $233 \mathrm{msec}$ lag) and the biological audiovisual condition (peak $\mathrm{r}=-.81$ at 200 msec lag).

The tight coupling between the temporal structure of movement and mu-ERD in the two conditions involving visual biological movement underscores the notion that this was a hMNS response and that it was primarily visually driven. The time lags observed (200-250 msec) are also within the range that might be expected between the onset of a visually observed motion stimulus and the onset of an event-related desynchronization response (see Rossi et al., 2014). Indirect evidence for sensitivity to the temporal structure of observed events has been found previously. For example, mu-ERD is stronger when observed actions follow a predictable sequence as compared to a scrambled sequence (e.g., Thomas et al., 2018). However, the current study is the first we are aware of that has linked the temporal structure of the mu-ERD response directly to the temporal structure in the stimuli.

In sum, the results of the current replication study are largely convergent with the findings of the two studies preceding it using the same stimuli. The two frontal sources identified (left vPMC [BA 6] and left anterior IFG [BA 47]) were corroborated as hMNS areas through inclusion of an action execution condition. Activation of these sources was influenced by the biologicalness of the stimuli (biological $>$ control) and the presentation modality (audiovisual = visual > audio). Unlike the prior studies, the current study found no evidence for multimodal enhancement of activation in either source. Exploratory analyses of mu-ERD in the vPMC for the visual and audiovisual biological conditions suggested that there was good tracking of the magnitude of movement in the stimulus.

\section{Declarations}

\section{Funding}

Natural Sciences and Engineering Research Council of Canada.

\section{Conflicts of interest/Competing interests}

The authors declare that they have no conflicts of interest.

\section{Ethics approval}

Approval was obtained from the Ryerson Ethics Board, reference number: REB 2010148.

\section{Consent to participate}

Informed consent was obtained from all individual participants included in the study. 


\section{Consent for publication}

Participants signed informed consent regarding confidential publishing of their data.

Availability of data, material, and code

Available upon request. 


\section{References}

Aflalo, T., Zhang, C. Y., Rosario, E. R., Pouratian, N., Orban, G. A., \& Andersen, R. A. (2020)A shared neural substrate for action verbs and observed actions in human posterior parietal cortex. Science advances, 6(43), eabb3984. doi:10.1126/sciadv.abb3984

Alhajri, N., Hodges, N. J., Zwicker, J. G., \& Virji-Babul, N. (2018). Mu suppression is sensitive to observational practice but results in different patterns of activation in comparison with physical practice. Neural Plasticity, 2018, 8309483-12. doi:10.1155/2018/8309483

Avenanti, A., Bolognini, N., Maravita, A., \& Aglioti, S. M. (2007). Somatic and motor components of action simulation. Current Biology, 17(24), 2129-2135. doi:10.1016/j.cub.2007.11.045

Babiloni, C., Babiloni, F., Carducci, F., Cincotti, F., Cocozza, G., Del Percuio, C., Moretti, D. V., \& Rossini, P. M. (2002). Human cortical encephalography (EEG) rhythms during the observation of simple aimless movements; a high-resolution EEG study. NeuroImage, 17(2), 559-572. doi:10.1006/nimg.2002.1192

Barbosa, A. V., Yehia, H. C., \& Vatikiotis-Bateson, E. (2008). Linguistically valid movement behavior measured non-invasively. In AVSP (pp. 173-177).

Bonini, L. (2017). The Extended Mirror Neuron Network: Anatomy, Origin, and Functions. The Neuroscientist, 23(1), 56-67. doi:10.1177/1073858415626400

Bookheimer, S. (2002). Functional MRI of language: New approaches to understanding the cortical organization of semantic processing. Annual Review of Neuroscience, 25(1), 151-188. doi:10.1146/annurev.neuro.25.112701.142946

Brucker, B., Ehlis, A.-C., Häußinger, F. B., Fallgatter, A. J., \& Gerjets, P. (2015). Watching corresponding gestures facilitates learning with animations by activating human mirror-neurons: an fNIRS study. Learn. Instr. 36, 27-37. doi: 10.1016/j.learninstruc.2014.11.003

Bruni, S., Bruni, S., Gerbella, M., Gerbella, M., Bonini, L., Bonini, L., Borra, E., Borra, E., Coudé, G., Coudé, G., Ferrari, P. F., Ferrari, P. F., Fogassi, L., Fogassi, L., Maranesi, M., Maranesi, M., Rodà, F., Rodà, F., Simone, L., . . . Rozzi, S. (2018). Cortical and subcortical connections of parietal and premotor nodes of the monkey hand mirror neuron network. Brain Structure \& Function, 223(4), 1713-1729. doi:10.1007/s00429-017-1582-0

Chartrand, T. L., \& van Baaren, R. B. (2009). Human mimicry. Advances in Experimental Social Psychology, 41, 219-274. doi:10.1016/S0065-2601(08)00405-X

Chong, T. T., Cunnington, R., Williams, M. A., Kanwisher, N., \& Mattingley, J. B. (2008). fMRI adaptation reveals mirror neurons in human inferior parietal cortex. Current Biology, 18(20), 1576-1580. doi:10.1016/j.cub.2008.08.068

Cochin, S., Barthelemy, C., Lejeune, B., Roux, S., \& Martineau, J. (1998). Perception of motion and qEEG activity in human adults. Electroencephalography and Clinical Neurophysiology, 107(4), 287-295. doi:10.1016/S0013-4694(98)00071-6 
Crawcour, S., Bowers, A., Harkrider, A., \& Saltuklaroglu, T. (2009). Mu wave suppression during the perception of meaningless syllables: EEG evidence of motor recruitment. Neuropsychologia, 47(12), 2558-2563.

Cuellar, M., Bowers, A., Harkrider, A. W., Wilson, M., \& Saltuklaroglu, T. (2012). Mu suppression as an index of sensorimotor contributions to speech processing: Evidence from continuous EEG signals. International Journal of Psychophysiology, 85(2), 242-248. doi:10.1016/j.ijpsycho.2012.04.003

Dean, I., Robinson, B. L., Harper, N. S., \& McAlpine, D. (2008). Rapid neural adaptation to sound level statistics. Journal of Neuroscience, 28(25), 6430-6438.

doi:10.1523/JNEUROSCI.0470-08.2008

Delorme, A., \& Makeig, S. (2004). EEGLAB: An open source toolbox for analysis of single-trial EEG dynamics including independent component analysis. Journal of Neuroscience Methods, 134(1), 9-21. doi:10.1016/j.jneumeth.2003.10.009

Delorme, A., Plamer, J., Oostenveld, R., Onton, J., \& Makeig, S. (2007). Comparing results of algorithms implementing blind source separation of EEG data. Swartz Foundation and NIH Grant.

di Pellegrino, G., Fadiga, L., Fogassi, L., Gallese, V., \& Rizzolatti, G. (1992). Understanding motor events: A neurophysiological study. Experimental Brain Research, 91(1), 176-180. doi:10.1007/BF00230027

Fadiga, L., Craighero, L., Buccino, G., \& Rizzolatti, G. (2002). Speech listening specifically modulates the excitability of tongue muscles: A TMS study. European Journal of Neuroscience, 15(2), 399-402. doi:10.1046/j.0953-816x.2001.01874.x

Filimon, F., Rieth, C. A., Sereno, M. I., \& Cottrell, G. W. (2015). Observed, executed, and imagined action representations can be decoded from ventral and dorsal areas. Cerebral Cortex (New York, N.Y. : 1991), 25(9), 3144-3158. doi:10.1093/cercor/bhu110

Fogassi, L., Ferrari, P. F., Gesierich, B., Rozzi, S., Chersi, F., \& Rizzolatti, G. (2005). Parietal lobe: From action organization to intention understanding. Science, 308(5722), 662-667. doi:10.1126/science. 1106138

Fox, N. A., Bakermans-Kranenburg, M. J., Yoo, K. H., Bowman, L. C., Cannon, E. N., Vanderwert, R. E., Ferrari, P. F., \& van IJzendoorn, M. H. (2016). Assessing human mirror activity with EEG mu rhythm: A meta-analysis. Psychological bulletin, 142(3), 291-313. doi:10.1037/bul0000031

Gallese, V., \& Goldman, A. (1998). Mirror neurons and the simulation theory of mind-reading. Trends in Cognitive Sciences, 2(12), 493-501. doi:10.1016/S1364-6613(98)01262-5

Gallese, V., Fadiga, L., Fogassi, L., \& Rizzolatti, G. (1996). Action recognition in the premotor cortex. Brain: A Journal of Neurology, 119 ( Pt 2)(2), 593-609. doi:10.1093/brain/119.2.593 
Gazzola, V., \& Keysers, C. (2009). The observation and execution of actions share motor and somatosensory voxels in all tested subjects: Single-subject analyses of unsmoothed fMRI data. Cerebral Cortex, 19(6), 1239-1255. doi:10.1093/cercor/bhn181

Hickok, G. (2010). The role of mirror neurons in speech perception and action word semantics. Language and Cognitive Processes: Cognitive Neuroscience of Language, 25(6), 749-776. doi:10.1080/01690961003595572

Hobson, H. M., \& Bishop, D. V. M. (2017). The interpretation of mu suppression as an index of mirror neuron activity: Past, present and future. Royal Society Open Science, 4(3), 160662. doi:10.1098/rsos. 160662

Holm, S. (1979). A simple sequentially rejective multiple test procedure.Scandinavian Journal of Statistics, 6(2), 65-70.

Horn, B. K., \& Schunck, B. G. (1981, November). Determining optical flow. In Techniques and Applications of Image Understanding (Vol. 281, pp. 319-331). International Society for Optics and Photonics.

Jeannerod, M. (2001). Neural simulation of action: A unifying mechanism for motor cognition. Neuroimage, 14(1), S103-S109. doi:10.1006/nimg.2001.0832

Kaplan, J.T., \& Iacoboni, M. (2007). Multimodal action representation in human left ventral premotor cortex. Cogn Process, 8, 103-113. doi:10.1007/s10339-007-0165-z

Kern, M., Ruescher, J., Schulze-Bonhage, A., \& Ball, T. (2019, March 27). Cortical MirrorSystem Activation During Real-Life Game Playing: An Intracranial Electroencephalography (EEG) Study. Retrieved June 17, 2020, from https://arxiv.org/abs/1902.09189

Keysers, C., Kohler, E., Umiltà, M. A., Nanetti, L., Fogassi, L., \& Gallese, V. (2003).

Audiovisual mirror neurons and action recognition. Experimental Brain Research, 153(4), 628636. doi:10.1007/s00221-003-1603-5

Kohler, E., Keysers, C., Umiltà, M. A., Fogassi, L., Gallese, V., \& Rizzolatti, G. (2002). Hearing sounds, understanding actions: Action representation in mirror neurons. Science, 297(5582), 846-848. doi:10.1126/science.1070311

Kuhlman, W. N. (1978). EEG feedback training of epileptic patients: Clinical and electroencephalographic analysis. Electroencephalography and Clinical Neurophysiology, 45(6), 699-710. doi:10.1016/0013-4694(78)90138-4

Lacadie, C. M., Fulbright, R. K., Constable, R. T., \& Papademetris, X. (2008). More accurate talairach coordinates for NeuroImaging using nonlinear registration. Neuroimage, 42(2), 717725. doi:10.1016/j.neuroimage.2008.04.240

Lakens, D., \& Caldwell, A. R. (2019). Simulation-based power-analysis for factorial ANOVA designs. doi:10.31234/osf.io/baxsf 
Lanzilotto, M., Ferroni, C. G., Livi, A., Gerbella, M., Maranesi, M., Borra, E., Passarelli, L., Gamberini, M., Fogassi, L., Bonini, L., \& Orban, G. A. (2019). Anterior intraparietal area: A hub in the observed manipulative action network. Cerebral Cortex (New York, N.Y. 1991), 29(4), 1816-1833. doi:10.1093/cercor/bhz011

Lanzilotto, M., Maranesi, M., Livi, A., Ferroni, C. G., Orban, G. A., \& Bonini, L. (2020). Stable readout of observed actions from format-dependent activity of monkey's anterior intraparietal neurons. Proceedings of the National Academy of Sciences - PNAS, 117(28), 16596-16605. doi:10.1073/pnas.2007018117

Lepage, J. F., \& Théoret, H. (2006). EEG evidence for the presence of an action observationexecution matching system in children. The European journal of neuroscience, 23(9), 25052510. doi:10.1111/j.1460-9568.2006.04769.x

Lévêque, Y., \& Schön, D. (2013). Listening to the human voice alters sensorimotor brain rhythms. PloS One, 8(11), e80659. doi:10.1371/journal.pone.0080659

MacQueen, J. B. (1967). Some methods for classification and analysis of multivariate observations. In L. M. Le Cam \& J. Neyman (Eds.), Proceedings of the fifth Berkeley symposium on mathematical statistics and probability (Vol. 1, pp. 281-297). California: University of California Press.

McGarry, L. M., Pineda, J. A., \& Russo, F. A. (2015). The role of the extended MNS in emotional and nonemotional judgments of human song. Cognitive, affective $\&$ behavioral neuroscience, 15(1), 32-44. doi:10.3758/s13415-014-0311-X

McGarry, L. M., Russo, F. A., Schalles, M. D., \& Pineda, J. A. (2012). Audiovisual facilitation of the mu rhythm. Experimental Brain Research, 218(4), 527-538. doi:10.1007/s00221-0123046-3

Miyakoshi, M. (2019, November 13). Makoto's preprocessing pipeline. 17.2.2. Retrieved June 16, 2020, from https://sccn.ucsd.edu/wiki/Makoto's_preprocessing_pipeline

Molnar-Szakacs, I., \& Overy, K. (2006). Music and mirror neurons: from motion to'e'motion. Social cognitive and affective neuroscience, 1(3), 235-241.

Mukamel, R., Ekstrom, A. D., Kaplan, J., Iacoboni, M., \& Fried, I. (2010). Single-neuron responses in humans during execution and observation of actions. Current biology: $\mathrm{CB}, 20(8)$, 750-756. doi:10.1016/j.cub.2010.02.045

Muthukumaraswamy, S. D., Johnson, B. W., \& McNair, N. A. (2004). Mu rhythm modulation during observation of an object-directed grasp. Brain research. Cognitive brain research, 19(2), 195-201. doi:10.1016/j.cogbrainres.2003.12.001

Naish, K. R., Houston-Price, C., Bremner, A. J., \& Holmes, N. P. (2014). Effects of action observation on corticospinal excitability: Muscle specificity, direction, and timing of the mirror response. Neuropsychologia, 64, 331-348. 
Neuper, C., Scherer, R., Wriessnegger, S., \& Pfurtscheller, G. (2009). Motor imagery and action observation: Modulation of sensorimotor brain rhythms during mental control of a braincomputer interface. Clinical Neurophysiology, 120(2), 239-247.

doi:10.1016/j.clinph.2008.11.015

Perry, A., \& Bentin, S. (2009). Mirror activity in the human brain while observing hand movements: a comparison between EEG desynchronization in the $\mu$-range and previous fMRI results. Brain Research, 1282, 126-132. doi:10.1016/j.brainres.2009.05.059

Perry, A., Stiso, J., Chang, E. F., Lin, J. J., Parvizi, J., \& Knight, R. T. (2018). Mirroring in the human brain: Deciphering the spatial-temporal patterns of the human mirror neuron system. Cerebral Cortex, 28(3), 1039-1048. doi:10.1093/cercor/bhx013

Pfurtscheller, G., Neuper, C., \& Krausz, G. (2000). Functional dissociation of lower and upper frequency mu rhythms in relation to voluntary limb movement. Clinical Neurophysiology, 111(10), 1873-1879. doi:10.1016/S1388-2457(00)00428-4

Pion-Tonachini, L., Kreutz-Delgado, K., \& Makeig, S. (2019). ICLabel: An automated electroencephalographic independent component classifier, dataset, and website. NeuroImage, 198, 181-197. doi:10.1016/j.neuroimage.2019.05.026

Pruitt, T. A., Halpern, A. R., \& Pfordresher, P. Q. (2019). Covert singing in anticipatory auditory imagery: Psychophysiology. Psychophysiology, 56(3), e13297. doi:10.1111/psyp.13297

R Core Team. (2019). R: A language and environment for statistical computing. R Foundation for statistical computing, Vienna, Austria. https://www.R-project.org.

Rizzolatti, G., \& Craighero, L. (2004). The mirror-neuron system. Annual Review of Neuroscience, 27(1), 169-192. doi:10.1146/annurev.neuro.27.070203.144230

Rizzolatti, G., Fadiga, L., Gallese, V., \& Fogassi, L. (1996a). Premotor cortex and the recognition of motor actions. Cognitive Brain Research, 3(2), 131-141. doi:10.1016/09266410(95)00038-0

Rizzolatti, G., Fogassi, L., \& Gallese, V. (2001). Neurophysiological mechanisms underlying the understanding and imitation of action. Nature Reviews Neuroscience, 2(9), 661-670. doi: $10.1038 / 35090060$

Rogalsky, C., Love, T., Driscoll, D., Anderson, S. W., \& Hickok, G. (2011). Are mirror neurons the basis of speech perception? evidence from five cases with damage to the purported human mirror system. Neurocase, 17(2), 178-187. doi:10.1080/13554794.2010.509318

Rossi, A., Parada, F. J., Kolchinsky, A., \& Puce, A. (2014). Neural correlates of apparent motion perception of impoverished facial stimuli: A comparison of ERP and ERSP activity. Neuroimage, 98, 442-459. doi:10.1016/j.neuroimage.2014.04.029 
Rozzi, S., Ferrari, P. F., Bonini, L., Rizzolatti, G., \& Fogassi, L. (2008). Functional organization of inferior parietal lobule convexity in the macaque monkey: Electrophysiological characterization of motor, sensory and mirror responses and their correlation with cytoarchitectonic areas. European Journal of Neuroscience, 28(8), 1569-1588. doi:10.1111/j.1460-9568.2008.06395.x

Rovetti, J., Copelli, F., \& Russo, F. A. (2021). The role of sensorimotor simulation in the recognition of speech emotion across different sensory modalities. Manuscript submitted for publication.

Russo, F. A. (2020). Motor system involvement in the perception of singing. In F. A. Russo, B. Ilari, \& A. J. Cohen (Eds I). The Routledge Companion to Interdisciplinary Studies in Singing: Volume I (pp. 276-288). Routledge.

Saygin, A. P., Wilson, S. M., Hagler, D. J., Jr, Bates, E., \& Sereno, M. I. (2004). Point-light biological motion perception activates human premotor cortex. Journal of Neuroscience, 24(27), 6181-6188. doi:10.1523/JNEUROSCI.0504-04.2004

SCCN. (2014, July 15). Chapter 05: Component Clustering Tools. Retrieved June 17, 2020, from https://sccn.ucsd.edu/wiki/Chapter_05:_Component_Clustering_Tools

Sterman, M. B., Macdonald, L. R., \& Stone, R. K. (1974). Biofeedback training of the sensorimotor electroencephalogram rhythm in man: effects on epilepsy. Epilepsia, 15(3), 395416. doi:10.1111/j.1528-1157.1974.tb04016.x

Talairach, J., \& Tournoux, P. (1988). Co-planar stereotaxic atlas of the human brain. Thieme, New York.

Thomas, R. M., De Sanctis, T., Gazzola, V., \& Keysers, C. (2018). Where and how our brain represents the temporal structure of observed action. NeuroImage (Orlando, Fla.), 183, 677-697.

Ulloa, E. R., \& Pineda, J. A. (2007). Recognition of point-light biological motion: Mu rhythms and mirror neuron activity. Behavioural Brain Research, 183(2), 188-194.

doi:10.1016/j.bbr.2007.06.007

Vigario, R., Sarela, J., Jousmiki, V., Hamalainen, M., \& Oja, E. (2000). Independent component approach to the analysis of EEG and MEG recordings. IEEE Transactions on Biomedical Engineering, 47(5), 589-593. doi:10.1109/10.841330

Wilson, S. M., \& Iacoboni, M. (2006). Neural responses to non-native phonemes varying in producibility: Evidence for the sensorimotor nature of speech perception. Neuroimage, 33(1), 316-325. doi:10.1016/j.neuroimage.2006.05.032

Yamazaki, Y., Yokochi, H., Tanaka, M., Okanoya, K., \& Iriki, A. (2010). Potential role of monkey inferior parietal neurons coding action semantic equivalences as precursors of parts of speech. Social Neuroscience, 5(1), 105-117. doi:10.1080/17470910802625306 